\title{
REPERCUSSÕES MATERNO-FETAIS DA ESTENOSE MITRAL NA GRAVIDEZ: UMA REVISÃO INTEGRATIVA
}

\section{MATERNAL-FETAL EFFECTS OF MITRAL STENOSIS DURING PREGNANCY: AN INTEGRATIVE REVIEW}

\section{EFECTOS MATERNO FETAL DE LA ESTENOSIS MITRAL DURANTE EL EMBARAZO: UNA REVISIÓN INTEGRATIVA}

\author{
Líscia Divana Carvalho Silva ${ }^{1}$, Dayane Conceição Monteiro ${ }^{2}$, Rafael de Abreu Lima ${ }^{3}$, \\ Cláudia Teresa Frias Rios ${ }^{4}$
}

\begin{abstract}
RESUMO
Objetivo: realizar uma revisão integrativa sobre as repercussões materno-fetais da estenose mitral na gravidez. Métodos: estudo realizado nas bases de dados Literatura LatinoAmericana e do Caribe em Ciências da Saúde, portal da Coordenação de Aperfeiçoamento de Pessoal de Ensino Superior e a Biblioteca Nacional de Medicina nas publicações de 2000 a 2013. A coleta de dados foi janeiro a fevereiro de 2014. Obteve-se uma amostra de nove publicações. Resultados: a estenose mitral apresenta maior repercussão materno-fetal em comparação a outras valvopatias. Repercussões maternas: edema pulmonar, hipertensão pulmonar, progressão de classe funcional, insuficiência cardíaca, aumento de medicamentos prescritos, taquicardia e a cardiomegalia. Repercussões fetais: retardo do crescimento fetal, aumento da taxa de prematuridade e baixo peso ao nascer. Conclusão: recomenda-se um trabalho integrado da equipe multiprofissional, terapêutica medicamentosa, controle dietético e restrição nas atividades cotidianas.
\end{abstract}

Descritores: Doenças Cardiovasculares; Estenose da Valva Mitral; Complicações Cardiovasculares na Gravidez.

\begin{abstract}
Objective: perform an integrative review about maternal-fetal repercussions of mitral stenosis during pregnancy. Methods: study on Literature Databases in Latin America and the Caribbean in Health Sciences, portal of Coordination of Improvement of Higher Education and the National Library of Medicine in 2000 to 2013 publications. Data collection was January to February 2014. Obtained a sample of nine publications. Results: the mitral stenosis presents greater maternal-fetal impact compared to other valvular heart diseases. The
\end{abstract}

\footnotetext{
1 Universidade Federal do Maranhão-UFMA. Centro de Ciências Biológicas e da Saúde. Professora do Departamento de Enfermagem-UFMA. Doutora em Ciências pela Escola de Enfermagem de Ribeirão PretoUniversidade de São Paulo (EERP-USP). Mestre em Enfermagem pela Universidade Federal do Ceará (UFC). Especialista em Cardiologia na Modalidade de Residência no Instituto Dante Pazzanese de Cardiologia (IDPC)São Paulo.

2 Universidade Federal do Maranhão-UFMA. Hospital Universitário. Ambulatório de Cardiologia. Enfermeira especialista em Cardiologia.

3 Universidade Federal do Maranhão-UFMA. Centro de Ciências Biológicas e da Saúde. Professor do Departamento de Enfermagem-UFMA.

4 Universidade Federal do Maranhão-UFMA. Centro de Ciências Biológicas e da Saúde. Professora do Departamento de Enfermagem-UFMA. Doutora em Saúde Coletiva (UFMA). Mestre em Enfermagem pela Universidade Federal do Ceará (UFC). Especialista em Enfermagem Obstétrica e Obstetrícia Social ((UFMA)
} 
maternal effects: pulmonary edema, pulmonary hypertension, progression of functional class, heart failure, increase in prescription drugs, tachycardia and cardiomegaly. The fetal effects: fetal growth, increased rate of prematurity and low birth weight. Conclusion: it is recommended that an integrated multidisciplinary team work, drug therapy, dietary control and restriction in daily activities.

Keywords: Cardiovascular Diseases; Mitral Valve Stenosis; Pregnancy Complications, Cardiovascular.

\section{RESUMEN}

Objetivo: realizar una revisión integrativa sobre repercusiones materno fetal de la estenosis mitral durante el embarazo. Métodos: estudio de bases de datos de la literatura en América Latina y el Caribe en Ciencias de la Salud, portal de Coordinación de Mejoramiento de la Educación Superior y la Biblioteca Nacional de Medicina entre 2000 al 2013.- Los datos fueron recolectados de enero a febrero de 2014. Se obtuvo una muestra de nueve publicaciones. Resultados: la estenosis mitral presenta mayor impacto materno fetal en comparación con otras enfermedades valvulares del corazón. Efectos maternos: edema pulmonar, hipertensión pulmonar, progresión de clase funcional, insuficiencia cardiaca, aumento de las medicinas recetadas, taquicardia y cardiomegalia. Consecuencias fetales: retraso de crecimiento fetal, aumento de la frecuencia de prematuridad y bajo peso al nacer. Conclusión: se recomienda que se trabaje en un equipo multidisciplinario integrado, terapia medicamentosa, control dietético y la restricción en las actividades cotidianas.

Palabras clave: Enfermedades Cardiovasculares; Estenosis de la Válvula Mitral; Complicaciones Cardiovasculares del Embarazo.

\section{INTRODUÇÃO}

A estenose mitral é determinada pela resistência ao fluxo sanguíneo do átrio para o ventrículo esquerdo, em razão do espessamento e da imobilidade dos folhetos. Destaca-se por ser a cardiopatia materna mais frequente, sendo responsável por 75 a $80 \%$ dos casos. ${ }^{1}$ A incidência média de cardiopatia na gravidez é de $4,2 \%$, oito vezes maior quando comparada às médias internacionais, sendo considerada a maior causa de morte materna indireta no ciclo gravídico-puerperal. ${ }^{2}$
A primeira manifestação clínica da estenose mitral pode ocorrer na gestação ou no puerpério imediato. ${ }^{3} \mathrm{~A}$ primeira avaliação cardiológica deve ser realizada antes da gravidez ou durante o primeiro trimestre, quando as alterações hemodinâmicas geralmente são sutis. Pacientes consideradas de baixo a médio riscos devem ser avaliadas novamente durante o final do segundo trimestre. ${ }^{4}$ Pacientes com alto risco requerem avaliações hemodinâmicas mais frequentes no periparto, pois até a amamentação, pode aumentar o trabalho cardíaco. Aquelas com estenose mitral grave devem ser seguidas por pelo menos seis meses após o parto 
para acompanhar a evolução clínica e avaliar o estado hemodinâmico. ${ }^{5}$ Para aquelas portadoras de estenose mitral com sinais de complicações obstétricas ou cardíacas, deve-se recomendar internação hospitalar. 6

A diferenciação de sinais e sintomas, entre gestantes normais e portadoras de cardiopatias torna-se muitas vezes difícil. A dispneia, o edema periférico e a fadiga, nesse período, podem não ser valorizados por serem comuns em gestantes normais no terceiro trimestre. Nas gestantes com estenose mitral são comuns as queixas de palpitação, tosse seca noturna, ortopnéia, dispneia paroxística noturna, hemoptise, dor precordial ao esforço, síncope. $^{3}$

O exame físico permite uma avaliação confiável, com alta especificidade para o diagnóstico das valvopatias e o conhecimento prévio de dados da história pode orientar o profissional de saúde durante a realização do exame físico (presença de sopro, ictus cordis desviado para a esquerda, hiperfonese e da primeira bulha cardíaca). Além dos achados semiológicos, exames de imagem como o ecocardiograma, tornam-se fundamentais por viabilizar a diferença das características patológicas (imagem valvar). O cateterismo cardíaco torna-se necessário quando há discrepância entre os achados clínicos, podendo fornecer informações adicionais para a tomada de decisões. ${ }^{6}$ A cirurgia cardíaca é indicada nos casos de congestão pulmonar refratária ao tratamento clínico ou na impossibilidade do tratamento percutâneo por cateter-balão. ${ }^{3}$

A estenose mitral além de apresentar elevada incidência, constitui-se numa das valvopatias de pior prognostico na gravidez, sendo considerada de alto risco. O conhecimento das repercussões da estenose mitral na gravidez é de fundamental importância para identificação precoce das complicações materno-fetais, estimativa do prognóstico e para a tomada de condutas, fornecendo subsídios para o planejamento de uma assistência mais qualificada. Este estudo objetiva realizar uma revisão integrativa sobre as repercussões materno-fetais da estenose mitral na gravidez.

\section{MÉTODO}

O processo de elaboração da revisão integrativa foi constituído por seis etapas: seleção de hipóteses ou questões para a revisão; seleção das pesquisas que irão compor a amostra da revisão; definição das características das pesquisas primárias que compõem a amostra; análise dos achados dos artigos incluídos na revisão; interpretação dos resultados e relato da revisão ${ }^{7}$, proporcionando um exame crítico dos achados. 
A formulação do problema se constituiu na seguinte questão norteadora: Qual conhecimento tem sido produzido sobre as repercussões da estenose mitral na gravidez?

O estudo foi realizado por meio das bases de dados: Literatura LatinoAmericana e do Caribe em Ciências da Saúde, portal da Coordenação de Aperfeiçoamento de Pessoal de Ensino Superior e a Biblioteca Nacional de Medicina. Foram utilizados os seguintes descritores: valvas cardíacas; estenose da valva mitral; gravidez, nos idiomas também em inglês e espanhol com os devidos cruzamentos. Foram incluídas publicações delineadas por algum critério metodológico, englobando artigos científicos, dissertações e teses publicadas no período de janeiro de 2000 a dezembro de 2013. O período de coleta de dados foi janeiro a fevereiro de 2014.

Na seleção das publicações, foi realizada a leitura criteriosa dos títulos e em seguida dos resumos para confirmar se respondiam a pergunta norteadora da pesquisa e em seguida realizava-se a leitura do artigo na íntegra para confirmação do atendimento aos critérios de inclusão e exclusão estabelecidos.

Ao realizar o cruzamento dos descritores identificou-se inicialmente 10.578 publicações. Na leitura dos títulos e resumos selecionou-se 8.798 publicações, destas após a leitura na íntegra selecionouse 45 publicações, sendo 25 (vinte e cinco) na base de dados do portal da Coordenação de Aperfeiçoamento de Pessoal de Ensino Superior, 12 (doze) na Literatura LatinoAmericana e do Caribe em Ciências da Saúde e 07 (sete) na Biblioteca Nacional de Medicina. Excluindo-se os estudos duplicados e publicados em duplicidade de idiomas, a amostra totalizou 09 (nove) publicações.

Esta pesquisa foi realizada de acordo com a Lei dos Direitos Autorais, que consiste a Lei $\mathrm{n}^{\circ} 9.610$ de fevereiro de 1998. ${ }^{8}$ Sendo assim, o estudo foi devidamente conduzido no sentido de não plagiar quaisquer trabalhos, sempre realizando devidamente a citação de fontes e autoria e dispensando tratamentos adequados aos dados obtidos.

\section{RESULTADOS}

As publicações selecionadas foram distribuídas pelos seguintes critérios: o ano das publicações, a origem, o tipo de estudo, os objetivos do estudo, o número amostral, o número de gestações, as complicações materno-fetais mais frequentes, os óbitos materno-fetais e as conclusões/recomendações dos autores.

O quadro 1 apresenta a distribuição das publicações segundo o primeiro autor, ano de publicação, origem, tipo e objetivos do estudo. 
Quadro 1: Distribuição das publicações segundo o autor, ano de publicação, origem, tipo de estudo e objetivos do estudo.

\begin{tabular}{|c|c|c|c|}
\hline $\begin{array}{l}\text { Primeiro } \\
\text { Autor/Ano }\end{array}$ & Origem & $\begin{array}{l}\text { Tipo } \\
\text { estudo }\end{array}$ & Objetivos \\
\hline $\begin{array}{l}\text { 1.Barbosa } \\
\text { PJB, 2000 }\end{array}$ & Brasil-BA & $\begin{array}{l}\text { Transversal, } \\
\text { analítico, } \\
\text { retrospectivo }\end{array}$ & $\begin{array}{l}\text { Identificar as características clínicas e } \\
\text { ecocardiográficas relacionadas com a ocorrência de } \\
\text { complicações no período da gestação e puerpério em } \\
\text { portadoras de estenose mitral }\end{array}$ \\
\hline $\begin{array}{l}\text { 2.Dessai, } \\
2000^{(10)}\end{array}$ & $\begin{array}{l}\text { Africa do } \\
\text { Sul- } \\
\text { Durban }\end{array}$ & $\begin{array}{l}\text { Transversal, } \\
\text { analítico, } \\
\text { prospectivo }\end{array}$ & $\begin{array}{l}\text { Avaliar a evolução da estenose mitral na gravidez, } \\
\text { prospectivamente, com ênfase nas mulheres com } \\
\text { sintomas persistentes. }\end{array}$ \\
\hline $\begin{array}{l}\text { 3.Hameed, } \\
2001^{(11)}\end{array}$ & $\begin{array}{l}\text { UA- Los } \\
\text { ngeles }\end{array}$ & $\begin{array}{l}\text { Caso controle, } \\
\text { longitudinal, } \\
\text { analítico, } \\
\text { retrospectivo, }\end{array}$ & $\begin{array}{l}\text { Avaliar a associação entre a doença valvular cardíaca } \\
\text { e as repercussões maternas e fetais através de uma } \\
\text { comparação com um grupo controle. }\end{array}$ \\
\hline $\begin{array}{l}\text { 4.Silverdis, } \\
2003^{(12)}\end{array}$ & $\begin{array}{l}\text { Canadá- } \\
\text { Toronto }\end{array}$ & $\begin{array}{l}\text { Coorte, } \\
\text { Longitudinal, } \\
\text { analítico, } \\
\text { prospectivo }\end{array}$ & $\begin{array}{l}\text { Definir os preditores de complicações cardíacas } \\
\text { maternas em mulheres grávidas com estenose mitral } \\
\text { reumática. Avaliar as alterações ecocardiográficas e } \\
\text { a gravidade da lesão durante a após a gravidez. }\end{array}$ \\
\hline $\begin{array}{l}\text { 5.Sobelga, } \\
2004^{(13)}\end{array}$ & $\begin{array}{l}\text { Polônia- } \\
\text { Cracóvia }\end{array}$ & $\begin{array}{l}\text { Transversal, } \\
\text { descritivo, } \\
\text { prospectivo }\end{array}$ & $\begin{array}{l}\text { Avaliar o desfecho da gravidez e do parto em } \\
\text { pacientes com doença cardíaca adquirida e após a } \\
\text { substituição da válvula, que estavam sob observação } \\
\text { no o Departamento de Doenças Cardiovasculares, } \\
\text { Instituto de Cardiologia, Cracóvia. }\end{array}$ \\
\hline $\begin{array}{l}\text { 6.Silva Shaah, } \\
2005^{(14)}\end{array}$ & ra- & Estudo & $\begin{array}{l}\text { Apresentar um estudo de caso sobre o impacto } \\
\text { fisiológico da sobrecarga volumétrica e da diurese na } \\
\text { pressão pulmonar no periparto de uma paciente com } \\
\text { estenose mitral moderada. }\end{array}$ \\
\hline $\begin{array}{l}\text { 7.Fernandes, } \\
2009^{(15)}\end{array}$ & $\begin{array}{l}\text { Brasil- } \\
\text { São Paulo }\end{array}$ & $\begin{array}{l}\text { Transversal, } \\
\text { descritivo, } \\
\text { retrospectivo }\end{array}$ & $\begin{array}{l}\text { Avaliar e descrever as principais repercussões } \\
\text { maternas (clínicas e obstétricas) e perinatais } \\
\text { relacionadas ao tipo predominante de lesão mitral } \\
\text { (estenose ou insuficiência) durante a gestação. } \\
\text { Avaliar a influencia das variáveis ecocardiográficas } \\
\text { sobre intercorrências maternas e perinatais }\end{array}$ \\
\hline $\begin{array}{l}\text { 8.Menachen, } \\
2011^{(16)}\end{array}$ & $\begin{array}{l}\text { EUA- } \\
\text { Nova } \\
\text { York }\end{array}$ & Estudo de caso & $\begin{array}{l}\text { Discutir o manejo do periparto de uma paciente com } \\
\text { estenose mitral severa segundo as repercussões } \\
\text { clínicas. }\end{array}$ \\
\hline $\begin{array}{l}\text { 9.Grant, } \\
2013^{(17)}\end{array}$ & $\begin{array}{l}\text { EUA- } \\
\text { Texas }\end{array}$ & Estudo de caso & $\begin{array}{l}\text { Apresentar um caso de parto complicado por } \\
\text { estenose mitral }\end{array}$ \\
\hline
\end{tabular}

Observa-se o maior número de publicações no ano de 2000 com 2 (duas) publicações, nos demais anos houve apenas 1 (uma) publicação. No que se refere ao tipo de estudo, identificou-se 4 (quatro) publicações na metodologia transversal (2 estudos analíticos e 2 descritivos), 2 (duas) publicações de natureza longitudinal (1 estudo de coorte prospectivo e 1 estudo caso controle 
retrospectivo) e 3 (três) publicações na forma de estudo de caso. Quanto aos objetivos das publicações analisadas, 6 (seis) publicações tinham como foco estudar especificamente a estenose mitral na gravidez. Uma publicação objetivou estudar a valvopatia mitral e suas repercussões materno-fetais e 2 (duas) publicações investigaram as lesões valvares na gestação, sendo feitas abordagens específicas sobre a estenose mitral.

Em relação à distribuição dos estudos segundo o número de mulheres, número de gestações e complicações materno-fetais, as repercussões maternas que se destacaram foram o edema pulmonar $(55,5 \%)$, a progressão de classe funcional $(33,3 \%)$, a hipertensão pulmonar severa $(22,2 \%)$, a insuficiência cardíaca congestiva $(22,2 \%)$, o aumento em torno de $11 \%$ na dose de medicamentos cardíacos prescritos, a taquicardia e a cardiomegalia. O crescimento intrauterino restrito foi a repercussão fetal mais frequente, correspondendo a 33,3\%, seguida da prematuridade $(11,1 \%)$ e baixo peso ao nascer $(11,1 \%)$. Em uma publicação o autor relatou não ter havido complicações fetais e em três publicações as repercussões fetais não foram descritas. Em relação a gravidade e desfecho das repercussões materno-fetais, duas publicações apresentaram percentual de morte materna que variou de $1,28 \%$ e $2,3 \%$ e quatro publicações descreveram o percentual de óbito fetal de $2,5 \%$ a $7,2 \%$.

A estenose mitral apresenta maior repercussão materno-fetal em comparação a outras valvopatias. A estenose mitral severa ou moderada e a história de eventos cardíacos antes da gravidez são preditores independentes de complicações maternas durante a gestação. Enfatiza-se que a área da valva mitral, a classe funcional, a hipertrofia e déficit da função ventricular esquerda são fatores de pior prognóstico. Os autores recomendam a importância de um trabalho integrado da equipe multiprofissional (Quadro 2).

Quadro 2: Distribuição das publicações segundo as conclusões e recomendações dos estudos.

\begin{tabular}{|l|l|}
\hline Publicação- Número & Conclusões/Recomendações \\
\hline Publicação 1 & $\begin{array}{l}\text { A área da valva mitral e a classificação funcional em gestantes } \\
\text { portadoras de estenose mitral são fatores prognósticos fortemente } \\
\text { associados a complicações maternas, entretanto não podem ser } \\
\text { associadas a eventos fetais/neonatais. }\end{array}$ \\
\hline Publicação 2 & $\begin{array}{l}\text { Os autores alertam para os prejuízos secundários ao diagnóstico } \\
\text { tardio bem como a falta de avaliação especificamente nos casos em } \\
\text { que a gestação ocorre em associação a estenose mitral, } \\
\text { comprometendo o curso saudável da gestação. }\end{array}$ \\
\hline
\end{tabular}




\begin{tabular}{|l|l|}
\hline Publicação 3 & $\begin{array}{l}\text { O estudo alerta para a necessidade de um acompanhamento } \\
\text { materno e fetal mais adequado e frequente. }\end{array}$ \\
\hline Publicação 4 & $\begin{array}{l}\text { O estudo aponta que a estenose mitral severa ou moderada e } \\
\text { historia de eventos cardíacos antes da gravidez são preditores } \\
\text { independentes de complicações maternas durante a gestação. }\end{array}$ \\
\hline Publicação 5 & $\begin{array}{l}\text { A presença de lesão estenótica severa, além do diagnóstico de } \\
\text { hipertrofia e déficit na função ventricular esquerda, são fatores de } \\
\text { risco elevado para a vida da gestante durante a gestação e após o } \\
\text { parto. }\end{array}$ \\
\hline Publicação 6 & $\begin{array}{l}\text { O estudo recomenda que o manejo seja feito de forma conservadora } \\
\text { para pacientes assintomáticas no intuito de minimizar a sobrecarga } \\
\text { volumétrica e prolongar o período diastólico. }\end{array}$ \\
\hline Publicação 7 & $\begin{array}{l}\text { Em comparação a insuficiência mitral, a estenose mitral apresentou } \\
\text { maior ocorrência de complicações maternas, obstétricas e } \\
\text { neonatais. Na avaliação das variáveis ecocardiográficas apenas a } \\
\text { área da valva mitral correlacionou-se o pior prognóstico no grupo } \\
\text { da estenose mitral. }\end{array}$ \\
\hline Publicação 8 & $\begin{array}{l}\text { O autor recomenda que o manejo das gestantes portadoras de } \\
\text { estenose mitral deve ser feito sobre um olhar multidisciplinar. }\end{array}$ \\
\hline Publicação 9 & $\begin{array}{l}\text { O autor destaca o resultado positivo que foi obtido diante do } \\
\text { encorajamento da gestante a levar adiante a gravidez, processo que } \\
\text { foi bem sucedido. }\end{array}$ \\
\hline
\end{tabular}

\section{DISCUSSÃO}

Dentre as valvopatias durante a gestação, a estenose mitral destaca-se por ser a mais comum. ${ }^{10,18}$ Apesar do desenvolvimento científico e tecnológico, o risco de complicações materno-fetais ainda permanece elevado. ${ }^{19-20}$ Isso pode ser explicado pela limitada adaptação às alterações cardiovasculares fisiológicas que ocorrem na gravidez, particularmente o aumento do volume plasmático e da frequência cardíaca. Além disso, o estado de hipercoagulabilidade aumenta o risco de tromboembolismo em pacientes com lesão mitral, fibrilação atrial e próteses valvares. A redução da resistência vascular periférica a partir do primeiro trimestre influencia na evolução das lesões valvares obstrutivas e em pacientes com hipertensão arterial sistêmica. $^{6}$

\section{A primeira manifestação} clínica da estenose mitral pode ocorrer frequentemente na gestação ou no puerpério imediato. As complicações maternas habituais são congestão pulmonar e arritmia supraventricular paroxística, e em menor frequência o tromboembolismo. ${ }^{3}$ A obstrução ao fluxo sanguíneo atrial gera um gradiente pressórico entre o átrio e o ventrículo esquerdos, e a elevação da pressão atrial esquerda transmite-se de maneira retrógrada ao leito vascular pulmonar, determinando congestão local, edema intersticial, hipertensão pulmonar e desenvolvimento progressivo de sintomas. ${ }^{1}$ 
Observou-se que dentre as 9 (nove) publicações selecionadas na presente pesquisa, 5 (cinco) publicações apresentaram o edema pulmonar como a complicação materna de maior incidência. Estudos identificam o edema pulmonar e a insuficiência cardíaca congestiva como as complicações maternas mais frequentes. ${ }^{9,20}$

A frequência de eventos fetais/neonatais está intimamente relacionada com a área da valva mitral e com a classificação funcional. ${ }^{21}$ A morbidade fetal tem sido estimada em aproximadamente $33 \%$ nas gestantes portadoras de estenose mitral severa, $28 \%$ nas pacientes com estenose mitral moderada e $14 \%$ nas pacientes com estenose mitral leve, sendo a prematuridade a principal causa independente da severidade da lesão. ${ }^{12} \mathrm{Um}$ estudo destacou a elevada taxa de crescimento intrauterino restrito em sua amostra. ${ }^{11}$ Em estudo prospectivo de 74 pacientes no Canadá observou uma maior representatividade de casos de prematuridade, não havendo nenhum tipo de intervenção invasiva para correção da estenose mitral. $^{21}$ No Brasil com uma amostra de 41 gestantes identificou-se que o baixo peso ao nascer foi a complicação neonatal mais frequente. $\mathrm{O}$ comprometimento hemodinâmico causado pela redução do fluxo sanguíneo imposto e as arritmias são prováveis explicações para a frequência elevada de restrição do crescimento fetal observada nos estudos. ${ }^{9}$ Além disso, medicamentos como diuréticos, digitálicos e betabloqueadores, têm sido associados com deficiência do fluxo sanguíneo uterino ou com o aumento da incidência de crescimento intrauterino restrito ou prematuridade. ${ }^{11}$

O surgimento dos sintomas se associa a um aumento da mortalidade. ${ }^{3} \mathrm{~A}$ estenose mitral associada à fibrilação atrial é uma condição clínica com grande risco de tromboembolismo sistêmico ${ }^{22}$, especialmente quando associados à insuficiência cardíaca geralmente têm prognóstico desfavorável na gravidez com maior risco de morbimortalidade e surgimento de complicações mais graves. ${ }^{23}$

Observou-se nesta revisão que apesar da morbidade ser elevada, a mortalidade materno-fetal teve frequência bastante limitada. ${ }^{12,24} \mathrm{O}$ diagnóstico precoce e o acompanhamento cuidadoso, possibilita maior restrição do número de óbitos, principalmente nos casos em que a mãe possui classe funcional I e II que são as mais prevalentes tendo seu prognóstico melhorado quando há tratamento adequado e eficaz. ${ }^{11}$ Os riscos da gravidez são maiores em pacientes em classe funcional III, que apresentam sobrecarga do ventrículo direito, fibrilação atrial e área valvar mitral reduzida. ${ }^{25}$ 


\section{CONCLUSÃO}

As gestantes portadoras de estenose mitral apresentam uma série de repercussões materno-fetais relacionadas a instabilidade hemodinâmica. As repercussões maternas identificadas por ordem decrescente foram o edema pulmonar, a progressão de classe funcional, a hipertensão da artéria pulmonar, a insuficiência cardíaca congestiva, o aumento da dose de medicamentos cardíacos prescritos, a taquicardia, a cardiomegalia. As repercussões fetais por ordem decrescente foram o retardo do crescimento fetal, o aumento da taxa de prematuridade e o baixo peso ao nascer.

Apesar da morbidade elevada, observou-se uma mortalidade maternofetal limitada, o percentual de morte materna variou de 1,28 a $2,3 \%$ e o percentual de óbito fetal de $2,5 \%$ a $7,2 \%$. Objetivando a redução das repercussões clínicas e o alívio dos sintomas, preconizase especialmente um tratamento medicamentoso com betabloqueadores e diuréticos, o controle dietético e a limitação nas atividades cotidianas.

O conhecimento e a identificação das repercussões maternofetais na gestante portadora de estenose mitral tornam-se fundamentais, sendo necessário o acompanhamento integrado por uma equipe multiprofissional para o planejamento de uma assistência qualificada. Como limitações do estudo, ressalta-se a escassez de publicações com ênfase na comparação sistemática entre as populações e estudos controlados randomizados com metodologias bem desenhadas para avaliar as repercussões da estenose na gravidez e as intervenções em saúde.

\section{REFERÊNCIAS}

1. Chandrashekhar Y, Westaby S, Ncarula J. Mitral stenosis. Lancet. 2009; 374(397):1271-83.

2. Wald RM, Siu SC. Heart Disease and Pregnancy. In: Yusuf S, Cairns JA, Camm AJ, Fallen EL, Gersh BJ, editors. Evidence-Based Cardiology. 3 ed. Oxford, UK: Wiley-Blackwell; 2010.

3. Avila WS, Freire CMV. Doença valvar. Arq Bras Cardiol. 2009; 93(6):1271-83.

4. Colman JM, Silversides CK, Semer M, Siu SC. Cardiac monitoring during pregnancy. In: Steer PJ, Gatzoulis MA, Baker P, editors. Heart disease and pregnancy. London: RCOG Press. 2006; 15(2):67-77.

5. Niwa K. Guidelines for Indication and Management of Pregnancy and Delivery in Women With Heart Disease (JCS 2010) Joint Working Groups. The Japanese Circulation Society, Japan Society of Obestetrics and Gynecology, Japanese Society of Pediatric Cardiology and Cardiac Surgery, The Japanese Society of Cardiovascular Surgery, Japanese College of Cardiology. Circ J. 2012; 76:240-60.

6. Tarasoutchi F, Montera MW, Grinberg M, Barbosa MR, Pinheiro DJ, Sánchez CRM, et al. Diretriz Brasileira de Valvopatias - SBC 2011 / I Diretriz Interamericana de Valvopatias - SIAC 2011. Arq Bras Cardiol. 2011; 97:1-67.

7. Mendes KDS, Silveira RCCP, Galvão C.M. Revisão integrativa: método de 
pesquisa para a incorporação de evidências na saúde e na enfermagem. Texto Contexto Enferm. 2008; 17(4):758-64.

8. Brasil. Lei no. 9.610, de 19 de fevereiro de 1998. Altera, atualiza e consolida a legislação sobre direitos autorais e dá outras providências. Diário Oficial [da República Federativa do Brasil, Brasília [online], 20 fev. 1998. Disponível em: http://www.planalto.gov.br/ccivil_03/leis/L 9610.htm

9. Barbosa PJB, Lopes AA, Feitosa GS, Almeida RVA, Mamédio RS, Brito, JC et al. Prognostic factors of rheumatic mitral stenosis during pregnancy and puerperium. Arq Bras Cardiol. 2000; 75:215-24.

10. Dessai DJP, Riveres AW, Price DL. A review of the current use of magnetic resonance imaging in pregnancy and safety implications for fetus. Prog Biophys Mol Biol. 2005; 87:335-53.

11. Hameed A, Karaalp IS, Tummala PP, Wani OR, Canetti M, Akhter MV et al. The effect of valvular heart disease on maternal and fetal outcome of pregnancy. $\mathrm{J}$ Am Coll Cardiol. 2001; 37:893-9.

12. Silverdis CK, Coman JM, Sermer M, Siu SC. Cardiac risk in pregnant women with rheumatic mitral stenosis. Am J Cardiol. 2003; 91:1382-5.

13. Sobelga LA, Tracz W, KostKiewicz M, Podolec P, Pasowicz M. Clinical and echocardiographic assessment of pregnant women with valvular heart diseasesmaternal and fetal outcome. International Journal Cardiology, 2004.

14. Shah SPM. Echocardiographic diagnosis of mitral valve prolapses. Journal of the American Society Echocardiography, 2005; 7: 286-93.

15. Fernandes A.F. Valvopatia mitral em gestantes: repercussões perinatais. Dissertação [Mestrado]. Faculdade de Medicina da Universidade de São Paulo (SP); 2009, 102 p.

16. Menaghen FT, Foster E, Glower DD, Kar S, Rinaldi MJ, Fail PS, et al.
Percutaneous repair or surgery for mitral regurgitation. N Engl J Med. 2011; 364(15):1395-406.

17. Grant EN, Williams KC, Perez BJ. Labor complicated by mitral stenosis. Proceedings Baylor University Medical Center. 2013; 26(1):42.

18. Salome N, Dias CC, Ribeiro J, Gonçalves M, Fonseca C, Ribeiro VG. Ballon mitral valvuloplasty during pregnancy-our experience. Rev Port Cardiol. 2002; 21(12):1437-44.

19. Stout KK, Otto CM. Pregnancy in women with valvular heart disease. Heart Journal. Washington, USA. 2007; 93:55258.

20. Bhatla N, Lal S, Behera G, Kriplani A, Mittal S, Agarwal N et al. Cardiac disease in pregnancy. Int $\mathrm{J}$ Gynaecol Obstet. 2003; 82:153-9.

21. Sawhney H, Aggarwal N, Suri V, Vasishta K, Sharma Y, Grover. A Maternal and perinatal outcome in rheumatic heart disease. Int J Gynaecol Obstet. 2003; 80:9 -14.

22. Avila WS, Gouveia AMM, Pomerantzeff P, Bortolloto MRL, Grinberg M, Stolf N, et al. Maternalfetal outcome and prognosis of cardiac surgery during pregnancy. Arq Bras Cardiol. 2009; 93(1):9-14.

23. Reimond SC, Rutherford JD. Clinical practice: valvular heart disease in pregnancy. N Engl J Med. 2003; 349:52-9.

24. Zelop C, Heffner LF. The downside of cesarean delivery: short- and longterm complications. Clin Obstet Gynecol. 2004; 47:386-93.

25. Lage EM, Barbosa AS. Cardiopatias e gravidez. Rev Feminina. 2012; 40(1):10-5.

Recebido em: 13/01/2016

Aprovado em: 12/07/2016

Publicado em: 31/07/2016 